EOMmun Communication et organisation

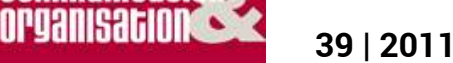

Les applications de la sémiotique à la communication des organisations

\title{
Chercheurs et praticiens: un panorama des modèles en communication des organisations
}

Laurent Morillon

OpenEdition

Journals

Édition électronique

URL : http://journals.openedition.org/communicationorganisation/3163

DOI : 10.4000/communicationorganisation.3163

ISSN : 1775-3546

Éditeur

Presses universitaires de Bordeaux

Édition imprimée

Date de publication : 1 juin 2011

Pagination : 229-240

ISBN : 978-2-86781-744-1

ISSN : $1168-5549$

Référence électronique

Laurent Morillon, « Chercheurs et praticiens: un panorama des modèles en communication des organisations ", Communication et organisation [En ligne], 39 | 2011, mis en ligne le 01 juin 2014 consulté le 01 mai 2019. URL : http://journals.openedition.org/communicationorganisation/3163 . DOI : 10.4000/communicationorganisation.3163 


\title{
Chercheurs et praticiens : un panorama des modèles en communication des organisations
}

\author{
Laurent Morillon'
}

Que ce soit au travers des injonctions faites aux scientifiques d'aller vers l'industrie et à celle-ci de contribuer au financement de la recherche, de l'intérêt des praticiens pour les recherches (afin d'améliorer leurs pratiques ou se distancier), de l'obligation faite aux chercheurs de vulgariser leur travaux... les occasions de rencontres, d'échanges, parfois de confrontations entre ces acteurs se multiplient. Ils se côtoient dans des colloques, des salles de cours, des journées d'études, des rencontres professionnelles, voire par l'entremise de supports de diffusion de connaissances (revue, ouvrage scientifique...) ou encore lors de thèses sous $\mathrm{CIFRE}^{2}$. Plus spécifiquement en ce qui concerne la communication des organisations ${ }^{3}$, le champ et les objets d'études induisent des liens entre chercheurs et praticiens qui peuvent s'avérer étroits. D'une part, les terrains peuvent être nécessaires à l'élaboration des connaissances, d'autre part, « les démarches de production des savoirs pour et dans les milieux professionnels se multiplient " (Bouzon, Meyer, 2008). Expertise, évaluation externe, consultance... les chercheurs ou praticiens/chercheurs sont invités à éclairer les conduites et les pratiques, à proposer un discours garant de l'acte et des connaissances opérationnalisables (Bouzon, Meyer, 2008).

Ce constat nous amène à questionner les rapports entre modèles théoriques et pratiques professionnelles dans le domaine de la communication des organisations. En cela, débuter par identifier les paradigmes épistémologiques des chercheurs et des praticiens nous paraît judicieux. Une recherche a été initiée pour recenser les modèles adoptés. Nous nous proposons de présenter ici une partie des résultats de deux travaux qui analysent le contenu de documents dépositaires. Le premier identifie les modèles en présence dans trente articles de revues scientifiques, le second dans six manuels qui traitent

1. Laurent Morillon est Maître de Conférences à l'Université Toulouse 3 et chercheur au LERASS. laurent. morillon@iut-tlse3.fr

2. Convention Industrielle de Formation par la REcherche.

3. Ce terme, choisi pour son caractère englobant, n'exclut pas les autres considérations de la communication (organisationnelle par exemple). 
en tout ou partie de communication des organisations. Le présent article nous donne l'occasion de croiser les modèles ainsi discernés.

Dans une première partie est légitimé l'intérêt d'une telle démarche. Dans une deuxième, l'approche par les documents est justifiée et la méthodologie mise en œuvre présentée. Une dernière partie propose une synthèse des résultats.

\section{Communication des organisations, quels modèles en présence ?}

Si la communication est depuis la révolution industrielle considérée comme un élément important de l'organisation, elle n'est objet scientifique que depuis le milieu du XX ${ }^{\mathrm{e}}$ siècle. Que ce soit en sciences de gestion, en sciences de l'information et de la communication (SIC) ou en sociologie, elle est aujourd'hui un objet de recherche à part entière (Bouzon, 2010 ; D'Almeida, Andonova, 2006). Dans les organisations, l'étude peut porter sur les politiques de communication, les processus, les moyens, les actes, les interactions ou encore les phénomènes cognitifs et sociaux associés (Bouzon, Meyer, 2006). Dans ce champ d'études, la frontière des univers des chercheurs et des praticiens s'avère particulièrement poreuse. Dans le cadre d'une recherche (et/ ou d'un enseignement pour les enseignant-chercheurs), l'organisation peut être un terrain d'études voire d'expérimentations. Les recherches peuvent devenir impliquées, appliquées voire des recherches actions. Dans le cadre de contrats signés avec des organisations, certains chercheurs réalisent ainsi des audits et contribuent à résoudre des problèmes de communication (Morillon, 2007). Au-delà d'une rétribution qui participe aux besoins financiers des laboratoires et parfois des chercheurs, ces actions permettent la production d'écrits universitaires censés assurer la reconnaissance de leurs auteurs (Heller, 1998).

Dans un tel contexte, questionner les rapports entre modèles théoriques et pratiques professionnelles dans le domaine de la communication des organisations apparaît opportun. Dans la mesure où la finalité d'une recherche est d'élaborer des connaissances, identifier les paradigmes épistémologiques adoptés est utile. Si ceux-ci sont aujourd'hui nombreux, oscillant schématiquement entre fonctionnalisme et interprétativisme (Bouzon, 2010), leur étude doit permettre de mieux connaitre les modes de définition et de construction de la connaissance et de participer à en juger la valeur. Réfléchir à ces questions s'impose d'autant plus que l'organisation est un creuset dans lequel se côtoient différentes disciplines. Or, si l'importation de concepts et de modèles est courante dans les interdisciplines, le champ de la communication des organisations intègre, souvent de manière allusive, des apports théoriques issus de la sociologie, de l'économie, des sciences de gestion, de la psychologie et la psychosociologie et de la linguistique.

Nous nous intéresserons plus particulièrement dans cet article aux modèles. Ceux-ci, issus de la «boîte à outils conceptuelle » (Mucchielli, Guivarch 1998) et choisis de manière ad hoc par le chercheur, dénotent son paradigme 
épistémologique. Ils ont « pour objet la représentation, soit le cadrage d'une réalité, permettant d'isoler et de comprendre un phénomène. Ils orientent donc les analyses en faisant apparaitre des éléments à mettre en relation » (Morillon, 2007). Quels sont les modèles adoptés par les chercheurs et ceux usités dans les pratiques professionnelles, notamment suite aux évolutions des théories des organisations (Rojot, 2003) ? Sans chercher à dresser un panorama exhaustif des modèles, le présent article rend compte de deux recherches menées, l'une sur des articles de revues scientifiques, l'autre sur des manuels.

\section{Recenser les modèles par l'entremise de documents}

Pour recenser les modèles nous nous proposons de considérer deux natures de documents dépositaires : les revues scientifiques et les manuels. Ils constituent selon nous une voie d'accès privilégiée à la construction collective du sens de l'activité mais aussi à la compréhension des processus de diffusion et de médiation des connaissances. La revue scientifique est aujourd'hui l'une des principales formes de communication érudite. Apparue au XVII ${ }^{e}$ siècle, elle est à notre époque constituée d'articles, soit autant de contributions évaluées (par un comité composé de chercheurs reconnus dans leur domaine) et publiées sous une forme normalisée (Devillard, Marco, 1993). Son rôle est de rassembler, rendre visible, diffuser, archiver et protéger la propriété intellectuelle de l'information scientifique au sein d'une communauté, mais également de la valider, contrôler, expertiser, évaluer, arbitrer et approuver par des pairs (Bégault, 2009). La mise en forme et la publication des résultats fait partie intégrante de la recherche : «le scientifique ne doit-il pas seulement faire la science, mais écrire la science (Day, 1989). Tant et si bien que la publication d'articles est devenue l'un des principaux critères d'évaluation des chercheurs. Merton, Garvey, Latour ont démontré le rôle fondamental de la communication scientifique dans le travail du chercheur. Les revues ont « un rôle de ciment théorique et une fonction fédératrice mais elles nous aident aussi, en tant qu'objet d'étude réflexif, à mieux comprendre les enjeux théoriques et sociaux qui gouvernent pour une part les états de la science » (Régimbeau, Couzinet, 2004).

Le manuel peut être défini comme " un ouvrage didactique ou scolaire qui expose les notions essentielles d'un art, d'une science, d'une technique ${ }^{4}$. Dans ceux qui traitent en tout ou partie de communication des organisations, la visée est praxéologique et la dimension instrumentale de la communication prédominante (D'Almeida, Andonova, 2006). Celle-ci est considérée comme un ensemble de méthodes et de techniques opératoires pour produire une valeur ajoutée immatérielle de nature cognitive, affective ou conative auprès de différents publics. Ces manuels proposent donc des outils et des modèles 
reproductibles d'actions à partir de théories. Cette production éditoriale est depuis plusieurs décennies (plus de vingt-cinq ans pour Publicitor) abondante. Certains ouvrages sont de taille conséquente (jusqu'à 700 pages), largement diffusés (150 000 exemplaires pour Mercator) et régulièrement réédités (cinquième édition pour Communicator). Ils sont utilisés dans les enseignements secondaire et supérieur professionnels, ainsi que dans les milieux de travail. Ils participent à la diffusion et à la reproduction de savoirs, de savoir-faire, de représentations et de schèmes structurants quant à la manière d'appréhender et gérer la communication. Leur performativité est potentiellement importante : "plusieurs centaines de milliers de "managers" dont certains exercent aujourd'hui des responsabilités influentes, ont donc été exposés aux manuels de Mc Carthy ou Kotler (1967) pour citer la première édition des plus célèbres, ou à toutes les versions traduites, adaptées voire plagiées de ces ouvrages (...) il sert de référence à la plupart des praticiens » (Marion, 1995).

$\mathrm{Au}$-delà de leurs rôles de "réservoir informationnel ", les manuels sont des artefacts cognitifs qui disposent d'une agentivité vis-à-vis des lecteurs en orientant certaines de leurs actions, la manière de les réaliser et en participant à la construction d'un sens. Ils sont également des objets de médiation ici des connaissances entre auteur(s) et lecteurs/co-auteurs qui font interagir un sujet avec un savoir à construire. Les discours sont en effet sélectionnés, contrôlés, organisés, orientés dans le temps de manière linéaire et conçus par un auteur en fonction d'un locuteur idéal (Maingueneau, 1998). Manuels et revues permettent donc de recenser les manières qu'ont les auteurs de formaliser et de présenter théories, modèles ou techniques. Ils permettent en outre d'accéder à des représentations de l'organisation et de la communication, mais aussi d'observer indirectement certaines des pratiques dans les milieux professionnels et scientifiques. Mais si ces documents contribuent à l'imposition, la naturalisation et la légitimation d'une vision ils sont avant tout des constructions sociales : le contenu n'est pas donné, ni transmis mais interprété, recontextualisé, complété et donc in fine reconstruit par des lecteurs à considérer comme autant de co-auteurs. En outre, les caractéristiques, significations, usages des documents évoluent avec les environnements, les points de vue et les logiques d'actions des individus qui les utilisent. Ainsi, pour peu qu'une démarche les construisent comme tels, ces documents peuvent être considérés comme des objets d'études scientifiques (Delcambre, 2000). Si les recherches sur les revues scientifiques existent, pour les manuels, hormis les travaux de Buttle (1995), Cameron et al. (2003) et Johansen \& Frandsen (1998), de telles réflexions semblent, à notre connaissance, assez rares notamment en France et relativement anciennes.

En matière de méthodologie, nous avons adopté une analyse de contenu sur un échantillon de manuels et d'articles de revues. À partir des modèles (explicitement ou non) exposés, des mots clefs utilisés et/ou des définitions 
adoptées, nous avons complété une grille d'analyse pré-catégorisée : paradigmes épistémologiques ${ }^{5}$, modèles, démarches scientifiques $6 . .$. Pour les articles, des analyses factorielles ont été menées à l'aide du logiciel Modalisa. Pour les manuels, l'analyse de contenu sémantique (sans logiciel) a été réalisée sur les parties dédiées à la théorie et/ou à l'exposition des modèles.

L'échantillon de documents est constitué d'auteurs de SIC et de sciences de gestion (SG). En communication des organisations, si le dialogue scientifique est limité, ces deux disciplines partagent certains objets scientifiques et/ ou projets (Courbet, 2001). Malaval affirme ainsi de manière quelque peu caricaturale dans son manuel : " La communication des entreprises et des organisations se trouve au croisement de deux champs théoriques : les sciences de la communication et les sciences de gestion. En effet, en tant que technique elle ne peut occulter les recherches développées depuis plus d'un demi-siècle sur la communication, mais elle ne peut pas non plus négliger sa finalité, à savoir être au service d'une stratégie d'entreprise » (Malaval et al., 2005). Notre travail est donc susceptible de recenser les divergences et convergences de paradigmes épistémologiques et de modèles entre ces disciplines. Il peut également « contribuer à favoriser un dialogue interdisciplinaire susceptible de fournir des réponses adaptées au monde professionnel et social » (Farchy et Froissart 2006).

Pour les articles scientifiques, trente articles francophones (quinze pour chaque discipline) publiés entre 1998 et 2010 sont retenus. Ils proviennent de treize revues ${ }^{7}$ classées par l'AERES ${ }^{8}$ et/ou les $\mathrm{CNU}^{9}$ des disciplines. Cet échantillon satisfait les critères de diversité, d'exhaustivité et de saturation sur quatre thèmes communs ${ }^{10}$. Afin de définir un corpus de travail raisonné parmi un nombre conséquent de manuels, nous avons retenu six d'entre eux $a$ priori les plus largement diffusés ${ }^{11}$, réédités et connus dans leur édition la plus récente : Communicator, Publicitor, Plan de Communication, Pentacom, Mercator et Marketing Management. Nous avons privilégié ceux dont le contenu, s'il est à finalité pratique et professionnelle, n'en est pas pour autant étayé " par des logiques strictement commerciales "vendre l'air du temps" » mais «par des références théoriques»(Bernard, 2003).

\footnotetext{
5. Positivisme, systémisme, constructivisme (Mucchielli, Guivarch, 1998) et interprétativisme (Giordano, 2001). 6. Descriptif, compréhensif, explicatif, prédictif (Evrard et al., 2003).

7. Revues en SG : Revue Française de Gestion (6 articles), Comptabilité, Contrôle, Audit (2), Décisions Marketing (1), Systèmes d'Information et Management (1), Finance, Contrôle, Stratégie (1), Management et Avenir (1), Entreprises et histoire (1), Recherche et Applications en Marketing (1), Gestion (1), Management International (1). Revues en SIC : Communication \& Organisation (6), Études de communication (3), Les Enjeux de l'information et de la communication (2), Communication et Langages (2), Communication (1).

8. Agence d'Evaluation de la Recherche et de l'Enseignement Supérieur.

9. Conseil National des Universités.

10. La Responsabilité Sociale de l'Entreprise (8 articles), le changement (9), les TIC (7) et la communication externe (6).

11. Cette diffusion est, sauf pour le Mercator (150 000 ex.), supputée par la réédition, faute de n'avoir obtenu le panel Livres Ipsos MediaCT.
} 
Certaines limites apparaissent. Le nombre assez restreint de manuels étudiés ne saurait permettre une généralisation des conclusions obtenues d'autant que des auteurs participent à plusieurs ouvrages ${ }^{12}$. Pour les revues, publier un article est « le moyen d'obtenir l'aval d'un comité de lecture qui garantit le niveau scientifique des travaux et permet d'être reconnu en tant que membre de sa communauté » (Begault, 2009). De fait, seuls les paradigmes épistémologiques les plus usuels de la discipline peuvent se trouver représentés. Cette contribution est donc à visée exploratoire. Nous projetons d'élargir nos échantillons et de mener des entretiens semi-directifs. Nous présentons ici une partie des résultats obtenus.

\section{Les modèles en présence, une synthèse des résultats}

Les résultats obtenus sont riches. Ils sont présentés de manière plus détaillée, mais non intégralement, dans deux communications (Morillon, Aldebert, Szafrajzen, 2010 ; Morillon, Gramaccia, 2011). Les contraintes éditoriales ne permettent de n'en aborder qu'une partie.

Pour les articles de revues scientifiques, nous nous centrons sur les résultats d'une analyse factorielle des correspondances liant épistémologie, méthodologie, problématique, démarche scientifique et discipline. Les articles sont positionnés dans un espace à plusieurs dimensions selon des similitudes de caractéristiques. Trois groupes d'articles assez homogènes apparaissent.

Le premier est constitué de textes, plutôt en SG, ancrés dans un paradigme positiviste, centrés sur l'explication et la détermination de causes et d'effets. Le raisonnement déductif et les méthodes quantitatives sont privilégiés. L'un des auteurs questionne par exemple le lien entre informatique et structure d'entreprise en utilisant une théorie de la contingence fortement déterministe. L'approche fonctionnaliste est prégnante. La communication, censée résoudre des problèmes, est rationalisée et instrumentalisée. Elle est " un canal » ${ }^{13}$, un « vecteur d'information », un « outil », un « support ». Elle permet de « diffuser les résultats de performance » ou encore d' « améliorer la circulation de l'information ». Ce premier groupe est cohérent avec l'histoire des SG et concorde, hormis pour la présence de modèles prédictifs, avec les constats de Courbet (2001). Les textes étudiés visent davantage à décrire les comportements et les processus mis en œuvre.

Le second groupe se compose plutôt d'auteurs SIC recherchant la compréhension et adoptant une approche systémique (par exemple " la négociation est intégrée dans une histoire non linéaire des relations, des interactions entre acteurs, des rapports de forces, des procédures de

12. Thierry Libaert est auteur de Plan de communication, co-auteur de Communicator et remercié pour ses conseils par l'éditeur du Pentacom. Des parties sont communes entre Mercator et Publicitor, Jacques Lendrevie participant aux deux.

13. Les références des citations issues des textes analysés ne sont volontairement pas indiquées mais peuvent nous être demandées. 
détermination, des lieux de discussion, des opérations de coordination, voire de coopération plus ou moins organisée »). Le raisonnement inductif est privilégié et les méthodes sont principalement qualitatives et/ou documentaires. Ces constats corroborent également ceux de Courbet (2001). Les auteurs tentent par des démarches empirico-inductives de décrypter la complexité, de comprendre les processus en prenant en compte temps et contexte.

Le troisième groupe comporte des textes des deux disciplines avec une visée descriptive et/ou prédictive. L'épistémologie est de convention constructiviste (Le Moigne, 2003) ${ }^{14}$ et le raisonnement privilégié abductif (par exemple « une organisation (...) émerge, se structure et se transforme à travers la réalisation de transactions entre le flot des conversations tenues et leur réification dans des textes »). En SG, les postures constructivistes se sont développées à la fin des années 1990 (Giordano, 2001). Dans l'un des textes, l'appréhension de cette co-construction des actions est posée : « le contrôle de la performance environnementale se construit sur des allers-retours entre la création de représentations formelles et les interprétations subjectives de celles-ci, notamment par les acteurs externes ». En SIC, la diffusion des approches communicationnelles des organisations favorise l'adoption de postures constructivistes : appréhender la communication comme " constituante de l'organisation ", un " espace de sens ", un moyen de « configurer une action ».

Concernant les manuels, le premier constat - que les ouvrages soient dédiés ou non à la communication et quelle que soit la discipline académique de leurs auteurs - est l'adoption d'un paradigme épistémologique positiviste parfois aménagé (Thiétart, 1999) : approche fonctionnaliste, raisonnements déductif et déterministe, "modèle marketing » (Mucchielli, Guivarch, 1998) sont en effet convoqués. Issu des sciences de gestion, ce dernier centre la communication sur la pratique et l'action. L'organisation et ses résultats sont au cœur des préoccupations. La communication est considérée comme une opération à piloter pour résoudre un problème. De fait, tous les ouvrages proposent une méthode basée schématiquement sur la définition d'une situation, la détermination d'objectifs à atteindre par la communication, l'identification de cibles, la mise en œuvre de moyens (dont un canal et un contenu), le contrôle des discours et l'évaluation des résultats. Ancré dans le courant de la sociologie des médias, le modèle marketing emprunte certains de ses principes au modèle de la communication à deux niveaux et à la théorie de l'information (Johansen, Frandsen, 1998). Confirmant les constats de Buttle en 1995, le modèle émetteur/récepteur (E/R) de Shannon et Weaver est systématiquement présenté de manière préliminaire ${ }^{15}$. Le message est « un stimulus destiné à être traité par l'appareil cognitif du destinataire chez

14. L'adoption commune d'une épistémologie constructiviste doit être relativisée. Les deux disciplines ne revendiquent par exemple pas le même socle théorique.

15. Sans que ses auteurs soient précisés dans Marketing Management (de même pour les " $5 \mathrm{~W}$ » de Laswell). Par ailleurs, seuls les auteurs de Pentacom qualifient explicitement le modèle E/R de positiviste. 
qui le stimulus va produire une réponse quelconque " (Johansen, Frandsen, 1998). Si le modèle $\mathrm{E} / \mathrm{R}$ apporte un vocabulaire de référence, il réduit également implicitement dans certains ouvrages la communication à la transmission d'informations (Dacheux, 2001) : « différents canaux à utiliser pour transmettre sa communication" (Kotler et al., 2006). Ce schéma est enrichi par la prise en compte d'une interaction avec le feed-back de Wiener et/ou par les « $5 \mathrm{~W}$ » de Lasswell. Les manuels dédiés à la communication peuvent y associer en plus les apports de l'école de Palo Alto, la capacité interprétative du destinataire, les approches systémique et/ou sémiotique. Les auteurs prennent donc appui sur des modèles canoniques simplificateurs et datés dont les limites sont connues mais dont la notoriété et la lisibilité sont appréciées des praticiens tout en accueillant des outils professionnels et/ou des apports théoriques plus ou moins récents ${ }^{16}$ de différentes disciplines (gestion, communication, éducation, politique, psychologie, sociologie...) dont certains contredisent lesdits modèles. Dans Communicator, quatre difficultés dont la communication souffrirait sont identifiées : vision mécaniste, approches quantitative, balistique et technique. Pourtant, les auteurs adoptent le modèle marketing ainsi qu'une définition construite en partie à partir du vocabulaire du schéma $\mathrm{E} / \mathrm{R}^{17}$. D'autres auteurs affirment des additions qui peuvent paraître surprenantes : «Il est possible de cumuler les modèle de Shannon, le principe de rétroaction, l'apport de la sémiotique et les messages de l'école de Palo-Alto. Cette démarche peut être mise en perspective avec le modèle des 5 W de Lasswell » (Malaval et al., 2005).

\section{Pour conclure}

Les liens entre chercheurs et praticiens ont fait l'objet d'un certain nombre de travaux. Viviane Couzinet a ainsi questionné les liens entre profession et recherche pour les sciences de l'information, dans leur composante documentation. Elle a notamment identifié ce qui rapproche, ce qui distingue et ce qui permet aux mondes professionnel et scientifique de se conforter mutuellement (Couzinet, 2003). Dans le domaine de la communication des organisations, les liens entre chercheurs et praticiens sont potentiellement nombreux. Les résultats des recherches menées, même s'ils ne sont pas présentés intégralement, sont susceptibles d'ouvrir de nombreux points de discussion (légitimité scientifique de l'addition de modèles a priori peu compatibles, instrumentalisation de la science, diversité des formes de constructivisme...). Nous proposons de conclure cet article en ne discutant que l'un d'entre eux, soit la nature des espaces de transition et d'interaction susceptibles de

\footnotetext{
16. Parfois de manière présomptueuse : «Intégrant les dernières avancées théoriques (...) la première partie (...) permet de présenter l'essentiel des théories de la communication et de ses fondements " (Malaval et al., 2005).

17. "La communication d'entreprise c'est l'action volontariste d'émission, de transmission et de réception de messages, dans un système de signes qui s'échangent au sein de l'entreprise et entre celle-ci et son environnement » (Westphalen, Libaert, 2009).
} 
répondre à la fois aux enjeux de la connaissance et à ceux de l'action. L'un de ces espaces peut-être ouvert par le modèle marketing, a priori commun à certains chercheurs et praticiens. Mais alors que les scientifiques explorent d'autres paradigmes, notamment constructivistes, comment expliciter leur absence dans les manuels ? Manipulation peu aisée dans l'action, opérationnalité limitée, techniques complexes et longues à mettre en œuvre, absence de « production de solutions immédiates, univoques, faciles d'emploi, vendables en quelques minutes à des comités de direction " (Augendre, 1997)... pour Mucchielli (2001) l'adoption d'une approche constructiviste dans les pratiques serait avant tout idéologique. Pourtant, si la plupart des praticiens, dans le cadre de leurs missions, sont en quête d'instruments et de modèles opérationnels, utiles et efficaces, susceptibles notamment de prédire les comportements, certains d'entre eux confrontés à la diversité des acteurs, au dynamisme des contextes ainsi qu'à la relative efficacité des modèles prédictifs s'ouvrent à d'autres approches. Constructivisme et systémisme, en permettant des analyses pluralistes des phénomènes en présence, apparaissent peu à peu dans certaines recherches-actions. L'un des auteurs du troisième groupe d'articles affirme ainsi : « il nous apparaît que l'approche constructionniste est la plus susceptible d'ouvrir de nouvelles pistes de recherches »; «Élaborer un modèle de la communication dans la mise en ouvre du changement. Inventorier les facteurs qui peuvent avoir une influence sur la communication du changement ». En s'inscrivant dans cette dynamique, il est possible de se demander par exemple dans quelle mesure les théories de la communication organisationnelle (Putnam, Nicotera, 2008) peuvent modifier les pratiques de communication interne.

\section{BIBLIOGRAPHIE}

ALMEIDA (D') N., ANDONOVA Y., " La communication des organisations », in OLIVESI S. (dir.), Sciences de l'information et de la communication. Objets, savoirs, discipline, Grenoble : PUG, 2006, pp. 129-143.

AUGENDRE M., «Les maux de la communication interne », in Sciences Humaines, hors série n¹6, mars/avril 1997.

BEGAULT B., «The scientific journal, a role for mediation of information between researches: what future in the digital environment? ", in RECIIS : Electronic Journal of Communication Information and Innovation in Health, vol.3, n 3, 2009.

BERNARD F., "La communication : enjeu de société, enjeu scientifique et enjeu de formation ", in Colloque national de Paris: Pour une refondation des enseignements de communication des organisations, Paris, 25-28 août 2003.

BOUZON A., " Entre communication et management. Evolution des fondements épistémologiques des organisations ", in II Congreso Internacional de la Asociación Española de Investigadores de la Comunicación (AE-IC), « Comunicación y desarrollo en 
la era digital », Faculté des sciences de la communication de 1'Université de Málaga, 3-5 Février 2010.

BOUZON A., MEYER V., La communication organisationnelle en question, méthodes et méthodologies, Paris : L'Harmattan, 2006.

BOUZON A., MEYER V., La communication des organisations entre recherche et action, Paris : L'Harmattan, 2008.

BUTTLE F.A., " Marketing communications theory: what do the texts teach our students? ", in International Journal of Advertising, Vol. 14, Blackwell Publishers Ltd., 1995, pp. 297-313.

CAMERON K.S., DUANE IRELAND R., LUSSIER R.N., RANDOLPH NEW J., ROBBIN S.P., " Management textbooks as propaganda ", in Journal of management education, 27, 6, dec. 2003, pp. 711-729.

COURBET D., "Comparaison épistémologique des recherches en SIC et sciences de gestion dans le domaine de la communication externe, divergences et terrain commun ", in Colloque La communication d'entreprise : regards croisés sciences de gestion et sciences de l'information et de la communication, Nice, 6-7 décembre 2001.

COUZINET V., «Praticiens de l'information et chercheurs : parcours, terrains et étayages ", in Documentaliste, sciences de l'information, 40(2), 2003, pp. 118-125.

DACHEUX E., « Etudier le marketing à la lumière de la communication », in L'année sociologique, 51, 2, 2001, pp. 411-427.

DAY R.A., How to Write and Publish a Scientific Paper, $3^{\mathrm{e}}$ éd., Cambridge : Cambridge University Press, 1989.

DELCAMBRE P., « Reconstruire des pratiques de communication à partir d' “objets”, documents d'archives ", in DELCAMBRE P. (coord), Communications organisationnelles, objets, pratiques, dispositifs. Rennes : PUR, 2000, pp. 205-222.

DEVILLARD J., MARCO L., Écrire et publier dans une revue scientifique, Paris : Les Editions d'Organisation, 1993.

EVRARD Y., PRAS B., ROUX E., Market : Etudes et recherches en marketing, Paris : Dunod, 2003.

FARCHY J., FROISSART P., « Le paradoxe de l'économie et de la communication », in Hermès, 44, 2006, pp. 9-18.

GIORDANO Y., "Les recherches en communication organisationnelle : du fonctionnalisme au constructivisme ", in MARTINET A-C., THIÉTART R-A. (coord.), Stratégies, Actualité et futurs de la recherche, Paris : Vuibert, 2001, pp. 159-174.

HELLER T., " Le chercheur face à la communication d'entreprise ", in LE MOËNNE C. (coord.), Communications d'entreprises et d'organisations, Rennes : Presses Universitaires de Rennes, 1998, pp. 13-26.

JOHANSEN W., FRANDSEN F., «Après le paradigme de Kotler, quel modèle pour la communication marketing ", in LE MOËNNE C. (coord.), Communications d'entreprises et d'organisations, Rennes : Presses Universitaires de Rennes, 1998, pp. 211-222.

KOTLER P., LANE KELLER K., DUBOIS B., MANCEAU D., Marketing management, $12^{\mathrm{e}}$ édition, Paris : Pearson Education, 2006. 
LE MOIGNE J.-L., Le constructivisme. Modéliser pour comprendre, Tome 3, Paris : L'Harmattan, 2003.

LENDREVIE J., DE BAYNAST A., EMPRIN C., Publicitor, $7^{\mathrm{e}}$ éd, Paris : Dunod, 2008.

LENDREVIE J., LÉVY J., LINDON D., Mercator, 8e éd., Paris : Dunod, 2006.

LIBAERT T., Le plan de communication : définir et organiser votre stratégie de communication, $3^{\mathrm{e}}$ éd., Paris : Dunod, 2008.

MAINGUENEAU D., Analyser les textes en communication, Paris : Dunod, 1998.

MAlaVAL P., DÉCAUdIN J-M., BÉNAROYA C., Pentacom, Paris : Pearson Education, 2005.

MARION G., "Le marketing management en question ", in Revue Française de Gestion, n¹02, janvier-février 1995, pp. 15-30.

MORILLON L., " Nomadisme du modèle marketing, quelle appropriation dans les recherches actions en communication organisationnelle ", in Communication E Organisation, $\mathrm{n}^{\circ} 31,2007$, pp. 215-227.

MORILLON L., ALDEBERT B., SZAFRAJZEN B., « Pour une lecture croisée des recherches sur la communication des organisations en sciences de l'information et de la communication et en sciences de gestion ", Dix-septième congrès de la SFSIC, Dijon, actes en ligne http://tabarqa.ubourgogne.fr/outils/OconfS/index.php/SIC/SFSIC17/paper/ view/88/8, 2010.

MORILLON L., GRAMACCIA G., " Pour une approche critique des modèles dans les manuels de communication des organisations ", in Colloque communication organisationnelle et pensée critique, Lille, 5-6 juillet 2011.

MUCCHIELLI A., La communication interne, les clefs d'un renouvellement, Paris : Armand Colin, 2001.

MUCCHIELLI A., GUIVARCH J., Nouvelles méthodes d'étude des communications, Paris : Armand Colin, 1998.

PUTNAM L.L., NICOTERA A.M., Building Theories of Organization: The Constitutive Role of Communication. New York : Routledge, 2008.

RÉGIMBEAU G., COUZINET V., « L'énonciation de la recherche en information-documentation : enjeux sociaux de la médiation des savoirs ", in Colloque international pluridisciplinaire Sciences et écritures, Besançon, 13-14 mai, Actes sur cédérom, 2004.

ROJOT J., Théorie des organisations, Paris : Eska, 2003.

THIETART R.A., Méthodes de recherche en management, Paris : Dunod, 1999.

WESTPHALEN M.-H., LIBAERT T., Communicator, 5e éd., Paris : Dunod, 2009.

Résumé : Les occasions de rencontres, d'échanges, parfois de confrontations entre chercheurs et praticiens se multiplient. En communication des organisations, le champ et les objets d'études induisent des liens qui peuvent s'avérer étroits. Nous nous proposons d'identifier les paradigmes épistémologiques et notamment les modèles en présence afin de questionner les rapports entre modèles théoriques et pratiques professionnelles. 
L'article présente une partie des résultats de deux recherches qui analysent le contenu de documents dépositaires : trente articles de revues scientifiques et six manuels.

Mots-clés : Épistémologie, modèle, communication des organisations, revue scientifique, manuel.

Abstract : Incentives and opportunities are growing for meetings, exchanges, and sometimes confrontations between researchers and practitioners. More specifically, regarding the communication of organisations, the scope and objects of study can create close links between us. We propose to identify epistemological paradigms and especially models, in order to question the relationship between theoretical models and professional practices. This article presents part of the results of two studies that analyze the content of certain documents: thirty articles from scientific journals and six textbooks.

Keywords : Epistemology, model, organizational communication, scientific reviews, bandbooks. 